\title{
Supplementary \\ How do hydrothermal liquefaction conditions and feedstock type influence product distribution and elemental composition?
}

René B. Madsen and Marianne Glasius

Interdisciplinary Nanoscience Center, Department of Chemistry, and Centre for Circular Bioeconomy, Aarhus University, Langelandsgade 140, Aarhus, Denmark

\section{A - Spirulina}

A.1. Prediction of bio-crude yield from HTL of Spirulina

Estimation of bio-crude yield from HTL of Spirulina at $345^{\circ} \mathrm{C}, 15 \mathrm{~min}$, and $12 \mathrm{wt} . \%$ using the model can be made by first converting the process conditions to factor levels.

$$
\begin{gathered}
345^{\circ} \mathrm{C}=1.5 \\
15 \min =-0.375 \\
12 \text { wt. } \%=-0.5
\end{gathered}
$$

The factor levels are then used in the quadratic model.

\begin{tabular}{|c|c|c|c|c|}
\hline & Gas - wt. $\%$ & Bio-crude - wt. \% & SR - wt. \% & AqP - wt. \% \\
\hline $270{ }^{\circ} \mathrm{C}, 10 \mathrm{~min}, 9$ wt. $\%$ & 9.0 & 27,3 & 9.4 & 54.3 \\
\hline $270^{\circ} \mathrm{C}, 10 \mathrm{~min}, 21$ wt. $\%$ & 8.0 & 25.6 & 10.3 & 56.1 \\
\hline $270^{\circ} \mathrm{C}, 26 \mathrm{~min}, 9$ wt. $\%$ & 9.6 & 26.3 & 2.4 & 61.7 \\
\hline $270^{\circ} \mathrm{C}, 26 \mathrm{~min}, 21 \mathrm{wt} . \%$ & 7.7 & 33.3 & 4.1 & 54.9 \\
\hline $330^{\circ} \mathrm{C}, 10 \mathrm{~min}, 9$ wt. $\%$ & 11.0 & 27.4 & 1.6 & 60.1 \\
\hline $330^{\circ} \mathrm{C}, 10 \mathrm{~min}, 21 \mathrm{wt} . \%$ & 14.3 & 36.9 & 1.2 & 47.6 \\
\hline $330^{\circ} \mathrm{C}, 26 \mathrm{~min}, 9 \mathrm{wt} . \%$ & 15.4 & 20.7 & 0.4 & 63.5 \\
\hline $330^{\circ} \mathrm{C}, 26 \mathrm{~min}, 21 \mathrm{wt} . \%$ & 17.4 & 37.0 & 0.4 & 45.2 \\
\hline $250{ }^{\circ} \mathrm{C}, 18 \mathrm{~min}, 15 \mathrm{wt} . \%$ & 7.4 & 30.0 & 6.6 & 56.0 \\
\hline $350{ }^{\circ} \mathrm{C}, 18 \mathrm{~min}, 15 \mathrm{wt} . \%$ & 17.4 & 34.9 & 2.0 & 45.7 \\
\hline $300{ }^{\circ} \mathrm{C}, 5 \mathrm{~min}, 15$ wt. $\%$ & 7.7 & 27.4 & 6.2 & 58.7 \\
\hline $300{ }^{\circ} \mathrm{C}, 31 \mathrm{~min}, 15$ wt. $\%$ & 13.5 & 34.2 & 1.6 & 50.7 \\
\hline $300{ }^{\circ} \mathrm{C}, 18 \mathrm{~min}, 5 \mathrm{wt} . \%$ & 12.8 & 31.7 & 0.0 & 55.5 \\
\hline $300{ }^{\circ} \mathrm{C}, 18 \mathrm{~min}, 25 \mathrm{wt} . \%$ & 15.5 & 38.0 & 1.8 & 44.7 \\
\hline $300{ }^{\circ} \mathrm{C}, 18 \mathrm{~min}, 15 \mathrm{wt} . \%$ & 11.6 & 38.4 & 4.0 & 47.0 \\
\hline
\end{tabular}

$$
\begin{aligned}
\text { bio }- \text { crude yield } & =38.5940+1.5 \times 1.2985-0.375 \times 0.8464-0.5 \times 3.0406+1.5 \times 0.375 \times 1.6491 \\
- & 1.5 \times 0.5 \times 2.5819+0.375 \times 0.5 \times 1.9181-1.5 \times 1.5 \times 2.7687-0.375 \times 0.375 \times 3.3736 \\
- & 0.5 \times 0.5 \times 1.9336=31.9013
\end{aligned}
$$

A.2. Experimental yields of gas, bio-crude, SR, and AqP from Spirulina

A.3. Experimental results of $\mathrm{CHNS}-\mathrm{O}$, carbon recovery, $\mathrm{HHV}$, and energy recovery of bio-crude from Spirulina

\begin{tabular}{|l|c|c|c|c|c|c|c|c|}
\hline & $\mathrm{C}[\%]$ & $\mathrm{H}[\%]$ & $\mathrm{N}[\%]$ & $\mathrm{S}[\%]$ & $\mathrm{O}[\%]$ & $\mathrm{C}_{\text {rec }}[\%]$ & $\mathrm{HHV}$ & ER [\%] \\
\hline $270{ }^{\circ} \mathrm{C}, 10 \mathrm{~min}, 9 \mathrm{wt} \%$ & 63.6 & 8.49 & 4.51 & 0.59 & 22.8 & 34.3 & 30.0 & 33.9 \\
\hline $270^{\circ} \mathrm{C}, 10 \mathrm{~min}, 21 \mathrm{wt} . \%$ & 62.5 & 8.10 & 6.64 & 0.56 & 22.2 & 31.6 & 29.4 & 31.2 \\
\hline $270^{\circ} \mathrm{C}, 26 \mathrm{~min}, 9 \mathrm{wt} \%$ & 67.3 & 7.81 & 6.58 & 0.69 & 17.6 & 35.0 & 31.3 & 34.1 \\
\hline $270^{\circ} \mathrm{C}, 26 \mathrm{~min}, 21 \mathrm{wt} \%$ & 66.4 & 7.93 & 7.25 & 0.61 & 17.8 & 43.7 & 31.1 & 42.8 \\
\hline $330^{\circ} \mathrm{C}, 10 \mathrm{~min}, 9 \mathrm{wt} \%$ & 68.2 & 7.90 & 6.29 & 0.83 & 16.8 & 36.9 & 31.8 & 36.0 \\
\hline $330^{\circ} \mathrm{C}, 10 \mathrm{~min}, 21 \mathrm{wt} \%$ & 67.2 & 7.94 & 7.41 & 0.77 & 16.6 & 48.9 & 31.6 & 48.1 \\
\hline $330^{\circ} \mathrm{C}, 26 \mathrm{~min}, 9 \mathrm{wt} \%$ & 70.1 & 8.14 & 6.06 & 0.70 & 15.0 & 28.7 & 32.9 & 28.2 \\
\hline $330^{\circ} \mathrm{C}, 26 \mathrm{~min}, 21 \mathrm{wt} \%$ & 69.8 & 8.21 & 6.68 & 0.73 & 14.6 & 51.0 & 33.0 & 50.4 \\
\hline
\end{tabular}




\begin{tabular}{|l|l|l|l|l|l|l|l|l|}
\hline $250{ }^{\circ} \mathrm{C}, 18 \mathrm{~min}, 15$ wt.\% & 61.6 & 7.91 & 6.69 & 0.67 & 23.1 & 36.6 & 28.8 & 35.8 \\
\hline $350{ }^{\circ} \mathrm{C}, 18 \mathrm{~min}, 15$ wt.\% & 70.9 & 8.34 & 6.53 & 0.73 & 13.6 & 48.9 & 33.6 & 48.5 \\
\hline $300{ }^{\circ} \mathrm{C}, 5 \mathrm{~min}, 15$ wt.\% & 62.6 & 7.57 & 6.62 & 0.73 & 22.5 & 33.9 & 28.8 & 32.7 \\
\hline $300{ }^{\circ} \mathrm{C}, 31 \mathrm{~min}, 15$ wt.\% & 70.2 & 8.23 & 6.84 & 0.66 & 14.1 & 47.4 & 33.2 & 46.9 \\
\hline $300{ }^{\circ} \mathrm{C}, 18 \mathrm{~min}, 5$ wt.\% & 68.9 & 7.92 & 6.35 & 0.85 & 15.9 & 43.2 & 32.2 & 42.2 \\
\hline $300{ }^{\circ} \mathrm{C}, 18 \mathrm{~min}, 25$ wt.\% & 67.4 & 7.92 & 7.53 & 0.76 & 16.4 & 50.6 & 31.6 & 49.7 \\
\hline $300^{\circ} \mathrm{C}, 18 \mathrm{~min}, 15$ wt.\% & 64.7 & 8.03 & 6.98 & 0.72 & 19.6 & 49.1 & 30.4 & 48.3 \\
\hline
\end{tabular}

\section{B-Miscanthus}

B.1. Experimental yields of gas, bio-crude, SR, and AqP from Miscanthus

\begin{tabular}{|c|c|c|c|c|}
\hline & Gas - wt. $\%$ & Bio-crude - wt. $\%$ & SR - wt. \% & AqP - wt. \% \\
\hline $270^{\circ} \mathrm{C}, 10 \mathrm{~min}, 9 \mathrm{wt} . \%$ & 4.7 & 29.0 & 50.6 & 15.7 \\
\hline $270^{\circ} \mathrm{C}, 10 \mathrm{~min}, 21$ wt. $\%$ & 6.3 & 35.5 & 46.1 & 12.1 \\
\hline $270{ }^{\circ} \mathrm{C}, 26 \mathrm{~min}, 9$ wt. $\%$ & 8.0 & 31.0 & 28.3 & 32.7 \\
\hline $270^{\circ} \mathrm{C}, 26 \mathrm{~min}, 21 \mathrm{wt} . \%$ & 11.7 & 39.7 & 28.2 & 20.4 \\
\hline $330{ }^{\circ} \mathrm{C}, 10 \mathrm{~min}, 9$ wt. $\%$ & 11.9 & 44.1 & 7.6 & 36.3 \\
\hline $330^{\circ} \mathrm{C}, 10 \mathrm{~min}, 21 \mathrm{wt} . \%$ & 16.2 & 50.7 & 11.1 & 22.0 \\
\hline $330^{\circ} \mathrm{C}, 26 \mathrm{~min}, 9 \mathrm{wt} . \%$ & 15.9 & 39.3 & 9.9 & 34.9 \\
\hline $330^{\circ} \mathrm{C}, 26 \mathrm{~min}, 21 \mathrm{wt} . \%$ & 21.3 & 46.0 & 9.8 & 22.8 \\
\hline $250^{\circ} \mathrm{C}, 18 \mathrm{~min}, 15$ wt. $\%$ & 7.2 & 28.0 & 49.1 & 15.7 \\
\hline $350{ }^{\circ} \mathrm{C}, 18 \mathrm{~min}, 15 \mathrm{wt} . \%$ & 20.7 & 40.2 & 10.7 & 28.4 \\
\hline $300{ }^{\circ} \mathrm{C}, 5 \mathrm{~min}, 15$ wt. $\%$ & 4.0 & 42.2 & 49.7 & 4.2 \\
\hline $300{ }^{\circ} \mathrm{C}, 31 \mathrm{~min}, 15 \mathrm{wt} . \%$ & 14.3 & 38.8 & 14.6 & 32.2 \\
\hline $300{ }^{\circ} \mathrm{C}, 18 \mathrm{~min}, 5$ wt. $\%$ & 11.8 & 38.8 & 13.3 & 36.1 \\
\hline $300{ }^{\circ} \mathrm{C}, 18 \mathrm{~min}, 25 \mathrm{wt} . \%$ & 17.6 & 46.3 & 14.4 & 21.7 \\
\hline $300{ }^{\circ} \mathrm{C}, 18 \mathrm{~min}, 15 \mathrm{wt} . \%$ & 13.6 & 44.6 & 13.9 & 27.9 \\
\hline
\end{tabular}

B.2. Experimental results of CHNS-O, carbon recovery, $\mathrm{HHV}$, and energy recovery of bio-crude from Miscanthus

\begin{tabular}{|l|c|c|c|c|c|c|c|c|}
\hline & $\mathrm{C}[\%]$ & $\mathrm{H}[\%]$ & $\mathrm{N}[\%]$ & $\mathrm{S}[\%]$ & $\mathrm{O}[\%]$ & $\mathrm{C}_{\text {rec }}[\%]$ & $\mathrm{HHV}$ & $\mathrm{ER}[\%]$ \\
\hline $270^{\circ} \mathrm{C}, 10 \mathrm{~min}, 9 \mathrm{wt} \%$ & 67.2 & 5.96 & 1.24 & 0.48 & 25.1 & 39.7 & 27.9 & 47.0 \\
\hline $270^{\circ} \mathrm{C}, 10 \mathrm{~min}, 21 \mathrm{wt} \%$ & 58.1 & 5.95 & 1.21 & 0.32 & 34.4 & 42.0 & 23.6 & 48.8 \\
\hline $270^{\circ} \mathrm{C}, 26 \mathrm{~min}, 9 \mathrm{wt} \%$ & 67.7 & 5.94 & 0.99 & 0.34 & 25.0 & 42.8 & 28.0 & 50.5 \\
\hline $270^{\circ} \mathrm{C}, 26 \mathrm{~min}, 21 \mathrm{wt} \%$ & 61.4 & 6.70 & 0.92 & 0.34 & 30.6 & 49.6 & 26.1 & 60.2 \\
\hline $330^{\circ} \mathrm{C}, 10 \mathrm{~min}, 9 \mathrm{wt} \%$ & 69.1 & 6.10 & 0.94 & 0.19 & 23.7 & 62.1 & 28.8 & 74.0 \\
\hline $330^{\circ} \mathrm{C}, 10 \mathrm{~min}, 21 \mathrm{wt} \%$ & 71.0 & 6.56 & 0.90 & 0.16 & 21.4 & 73.3 & 30.3 & 89.3 \\
\hline $330^{\circ} \mathrm{C}, 26 \mathrm{~min}, 9 \mathrm{wt} \%$ & 74.1 & 6.19 & 1.13 & 0.15 & 18.4 & 59.3 & 31.3 & 71.5 \\
\hline $330^{\circ} \mathrm{C}, 26 \mathrm{~min}, 21 \mathrm{wt} \%$ & 74.7 & 6.69 & 1.02 & 0.13 & 17.5 & 70.0 & 32.2 & 86.1 \\
\hline $250^{\circ} \mathrm{C}, 18 \mathrm{~min}, 15 \mathrm{wt} \%$ & 63.2 & 5.67 & 1.16 & 0.16 & 29.8 & 36.0 & 25.6 & 41.7 \\
\hline $350^{\circ} \mathrm{C}, 18 \mathrm{~min}, 15 \mathrm{wt} \%$ & 73.3 & 6.74 & 1.04 & 0.13 & 18.8 & 60.1 & 31.6 & 73.9 \\
\hline $300^{\circ} \mathrm{C}, 5 \mathrm{~min}, 15 \mathrm{wt} \%$ & 54.9 & 5.80 & 1.16 & 0.23 & 37.9 & 47.2 & 21.9 & 53.8 \\
\hline $300^{\circ} \mathrm{C}, 31 \mathrm{~min}, 15 \mathrm{wt} \%$ & 71.0 & 6.34 & 0.86 & 0.11 & 21.7 & 56.1 & 30.0 & 67.7 \\
\hline $300^{\circ} \mathrm{C}, 18 \mathrm{~min}, 5 \mathrm{wt} \%$ & 70.0 & 6.02 & 0.96 & 0.10 & 22.9 & 55.3 & 29.1 & 65.8 \\
\hline $300^{\circ} \mathrm{C}, 18 \mathrm{~min}, 25 \mathrm{wt} \%$ & 67.3 & 6.64 & 0.87 & 0.10 & 25.1 & 63.5 & 28.7 & 77.2 \\
\hline $300^{\circ} \mathrm{C}, 18 \mathrm{~min}, 15 \mathrm{wt} \%$ & 67.0 & 6.24 & 0.95 & 0.10 & 25.7 & 60.7 & 28.0 & 72.4 \\
\hline
\end{tabular}




\section{C - Sewage sludge}

C.1. Experimental yields of gas, bio-crude, $\mathrm{SR}$, and AqP from sewage sludge

\begin{tabular}{|c|c|c|c|c|}
\hline & Gas - wt. $\%$ & Bio-crude - wt. $\%$ & SR - wt. \% & AqP - wt. \% \\
\hline $270{ }^{\circ} \mathrm{C}, 10 \mathrm{~min}, 9$ wt. $\%$ & 5.6 & 29.7 & 55.8 & 8.9 \\
\hline $270{ }^{\circ} \mathrm{C}, 10 \mathrm{~min}, 21$ wt. $\%$ & 4.9 & 33.9 & 52.3 & 8.9 \\
\hline $270{ }^{\circ} \mathrm{C}, 26 \mathrm{~min}, 9$ wt. $\%$ & 9.4 & 33.0 & 30.2 & 27.4 \\
\hline $270^{\circ} \mathrm{C}, 26 \mathrm{~min}, 21 \mathrm{wt} \%$ & 9.8 & 35.6 & 34.6 & 20.0 \\
\hline $330{ }^{\circ} \mathrm{C}, 10 \mathrm{~min}, 9$ wt. $\%$ & 13.2 & 42.2 & 15.9 & 28.6 \\
\hline $330^{\circ} \mathrm{C}, 10 \mathrm{~min}, 21 \mathrm{wt} . \%$ & 13.9 & 39.5 & 20.2 & 26.3 \\
\hline $330{ }^{\circ} \mathrm{C}, 26 \mathrm{~min}, 9 \mathrm{wt} . \%$ & 15.2 & 38.5 & 17.5 & 28.8 \\
\hline $330^{\circ} \mathrm{C}, 26 \mathrm{~min}, 21 \mathrm{wt} . \%$ & 19.3 & 38.8 & 20.1 & 21.9 \\
\hline $250^{\circ} \mathrm{C}, 18 \mathrm{~min}, 15 \mathrm{wt} . \%$ & 8.0 & 31.2 & 42.1 & 18.6 \\
\hline $350{ }^{\circ} \mathrm{C}, 18 \mathrm{~min}, 15 \mathrm{wt} . \%$ & 17.1 & 37.7 & 18.0 & 27.2 \\
\hline $300{ }^{\circ} \mathrm{C}, 5 \mathrm{~min}, 15 \mathrm{wt} . \%$ & 5.7 & 36.9 & 44.3 & 13.1 \\
\hline $300^{\circ} \mathrm{C}, 31 \mathrm{~min}, 15$ wt. $\%$ & 15.9 & 35.1 & 21.2 & 27.7 \\
\hline $300{ }^{\circ} \mathrm{C}, 18 \mathrm{~min}, 5 \mathrm{wt} . \%$ & 10.5 & 38.9 & 14.5 & 36.2 \\
\hline $300{ }^{\circ} \mathrm{C}, 18 \mathrm{~min}, 25 \mathrm{wt} . \%$ & 16.5 & 39.7 & 20.7 & 23.1 \\
\hline $300{ }^{\circ} \mathrm{C}, 18 \mathrm{~min}, 15 \mathrm{wt} . \%$ & 14.3 & 41.5 & 18.7 & 25.5 \\
\hline
\end{tabular}

C.2. Experimental results of CHNS-O, carbon recovery, $\mathrm{HHV}$, and energy recovery of bio-crude from sewage sludge

\begin{tabular}{|c|c|c|c|c|c|c|c|c|}
\hline & $\mathrm{C}[\%]$ & $\mathrm{H}[\%]$ & $\bar{N}[\%]$ & S [\%] & $\mathrm{O}[\%]$ & $\mathrm{C}_{\mathrm{rec}}[\%]$ & $\mathrm{HHV}$ & ER [\%] \\
\hline $270{ }^{\circ} \mathrm{C}, 10 \mathrm{~min}, 9$ wt. $\%$ & 67.0 & 9.57 & 2.27 & 1.12 & 20.0 & 42.8 & 32.7 & 42.9 \\
\hline $270{ }^{\circ} \mathrm{C}, 10 \mathrm{~min}, 21$ wt. $\%$ & 69.6 & 9.69 & 3.02 & 1.17 & 16.6 & 50.8 & 34.2 & 44.8 \\
\hline $270^{\circ} \mathrm{C}, 26 \mathrm{~min}, 9 \mathrm{wt} . \%$ & 74.5 & 10.4 & 1.87 & 1.07 & 12.2 & 52.9 & 37.1 & 48.7 \\
\hline $270{ }^{\circ} \mathrm{C}, 26 \mathrm{~min}, 21 \mathrm{wt} . \%$ & 71.8 & 9.87 & 2.42 & 1.05 & 14.9 & 55.0 & 35.3 & 46.3 \\
\hline $330^{\circ} \mathrm{C}, 10 \mathrm{~min}, 9 \mathrm{wt} . \%$ & 75.3 & 9.88 & 1.94 & 1.06 & 11.8 & 68.4 & 36.9 & 48.3 \\
\hline $330{ }^{\circ} \mathrm{C}, 10 \mathrm{~min}, 21 \mathrm{wt} . \%$ & 72.7 & 9.71 & 2.40 & 1.00 & 14.1 & 61.9 & 35.5 & 46.6 \\
\hline $330{ }^{\circ} \mathrm{C}, 26 \mathrm{~min}, 9 \mathrm{wt} . \%$ & 74.2 & 9.86 & 2.24 & 1.06 & 12.7 & 61.4 & 36.4 & 47.7 \\
\hline $330^{\circ} \mathrm{C}, 26 \mathrm{~min}, 21 \mathrm{wt} . \%$ & 75.2 & 9.10 & 3.03 & 0.97 & 11.7 & 62.7 & 36.0 & 47.2 \\
\hline $250{ }^{\circ} \mathrm{C}, 18 \mathrm{~min}, 15 \mathrm{wt} . \%$ & 72.3 & 9.93 & 2.41 & 1.12 & 14.2 & 48.5 & 35.7 & 46.7 \\
\hline $350{ }^{\circ} \mathrm{C}, 18 \mathrm{~min}, 15 \mathrm{wt} . \%$ & 73.6 & 9.36 & 2.84 & 1.10 & 13.1 & 59.6 & 35.6 & 46.6 \\
\hline $300{ }^{\circ} \mathrm{C}, 5 \mathrm{~min}, 15$ wt. $\%$ & 60.3 & 9.05 & 2.84 & 1.03 & 26.8 & 47.9 & 29.0 & 38.1 \\
\hline $300{ }^{\circ} \mathrm{C}, 31 \mathrm{~min}, 15 \mathrm{wt} . \%$ & 75.4 & 9.62 & 2.56 & 1.04 & 11.4 & 56.9 & 36.7 & 48.1 \\
\hline $300^{\circ} \mathrm{C}, 18 \mathrm{~min}, 5 \mathrm{wt} . \%$ & 75.9 & 10.3 & 1.42 & 0.97 & 11.4 & 63.4 & 37.6 & 49.3 \\
\hline $300{ }^{\circ} \mathrm{C}, 18 \mathrm{~min}, 25 \mathrm{wt} . \%$ & 73.4 & 9.43 & 2.69 & 0.93 & 13.6 & 62.7 & 35.5 & 46.5 \\
\hline $300{ }^{\circ} \mathrm{C}, 18 \mathrm{~min}, 15 \mathrm{wt} . \%$ & 72.8 & 9.71 & 2.46 & 1.01 & 14.0 & 65.0 & 35.6 & 46.6 \\
\hline
\end{tabular}

